\title{
CDX2 expression in the intestinal-type gastric epithelial neoplasia: frequency and significance
}

\author{
Do Youn Park ${ }^{1,2}$, Amitabh Srivastava ${ }^{3}$, Gwang Ha Kim ${ }^{4,5}$, Mari Mino-Kenudson ${ }^{1}$, \\ Vikram Deshpande $^{1}$, Lawrence R Zukerberg ${ }^{1}$, Geum Am Song ${ }^{4,5}$ and Gregory Y Lauwers ${ }^{1}$ \\ ${ }^{1}$ Department of Pathology, Massachusetts General Hospital, Boston, MA, USA; ${ }^{2}$ Department of Pathology, \\ Pusan National University Hospital, Busan, Korea; ${ }^{3}$ Department of Pathology, Dartmouth Hitchcock Medical \\ Center and Dartmouth Medical School, Lebanon, NH, USA; ${ }^{4}$ Department of Gastroenterology, Pusan National \\ University Hospital, Busan, Korea and ${ }^{5}$ Medical Research Institute, Pusan National University, Busan, Korea
}

\begin{abstract}
CDX2 is an intestinal transcription factor responsible for regulating the proliferation and differentiation of intestinal epithelial cells. In gastric adenocarcinoma, CDX2 expression is known to be associated with limited invasiveness and intestinal phenotypes. The aims of this study were to analyze CDX2 expression in a series of well-characterized cases of gastric epithelial dysplasia, based on the morphologic and mucin phenotypes, and also to analyze CDX2 expression along the metaplasia-dysplasia-carcinoma sequence. CDX2 expression was evaluated in 69 cases of gastric epithelial dysplasia, 88 cases of intestinal-type early gastric cancers, and 56 cases of advanced gastric cancers. Increased CDX2 expression was more frequently associated with adenomatous-type gastric epithelial dysplasia $(27 / 31,87 \%)$ compared with foveolar $(7 / 15,47 \%)$ or hybrid $(10 / 23,44 \%)$ types of gastric epithelial dysplasia $(P=0.001)$. CDX2 expression correlated with an increase in CD10 expression $(P=0.005)$, and a decrease in MUC5AC expression $(P=0.001)$ in gastric epithelial dysplasia. CDX2 expression was also gradually decreased from gastric epithelial dysplasia, to early and advanced gastric cancers (present in 64,40 and $27 \%$ of the cases, respectively). A negative correlation was also observed between CDX2 expression and the depth of tumor invasion. Our results indicate that CDX2 expression is associated with specific morphological and mucin phenotypes of gastric epithelial dysplasias, and decreases progressively with the advancing stage of gastric cancers, suggesting a possible tumor suppressor role for CDX2.
\end{abstract}

Modern Pathology (2010) 23, 54-61; doi:10.1038/modpathol.2009.135; published online 9 October 2009

Keywords: CDX2; dysplasia; gastric cancer; mucin

Gastric adenocarcinoma is broadly divided into intestinal and diffuse types, and remains one of the most prevalent malignant tumors worldwide. ${ }^{1,2}$ The pathogenesis of the intestinal type of gastric cancer is closely linked with well-defined precursors such as chronic atrophic gastritis, intestinal

Correspondence: Dr GY Lauwers, MD, Department of Pathology, Massachusetts General Hospital, Gastrointestinal Pathology Service, 55 Fruit Street, WRN 2, Boston, MA 02114-2696, USA.

E-mail: glauwers@partners.org

Presented in part at the annual meeting of United States and Canadian Academy of Pathology, San Diego, CA, 24-30 March 2007. This study was supported by a grant from the National R\&D Program for Cancer Control, Ministry for Health, Welfare and Family affairs, Republic of Korea (Project ID: 0920050).

Received 2 December 2008; revised 19 May 2009; accepted 20 May 2009; published online 9 October 2009 metaplasia, and adenoma/dysplasia, whereas the pre-malignant lesions of sporadic diffuse-type gastric cancer are not well defined. ${ }^{1,3}$ Furthermore, despite wide acceptance of the gastritis-metaplasiadysplasia-carcinoma sequence, the precise molecular alterations underlying this progression pathway remain to be delineated. ${ }^{4}$

CDX2 is a Drosophila caudal-related homeobox transcription factor responsible for early intestinal differentiation. ${ }^{5,6}$ CDX2 may stimulate intestinal proliferation and differentiation, by transcriptional activation of intestine-specific proteins (MUC2, sucrase-isomaltase, carbonic anhydrase I), or act as a growth inhibitor through activation of WAF1 (cyclin-dependent kinase inhibitor). ${ }^{7,8}$ Several reports have also suggested a tumor suppressor role for CDX2 in human colorectal carcinogenesis, and 
this might also hold true for gastric cancers. ${ }^{6,9-11}$ Previous studies also suggest that CDX2 expression is associated not only with an intestinal mucin phenotype but also with a less invasive phenotype, and thus an early stage of gastric cancer. ${ }^{12-14}$ However, there are scant and conflicting data about CDX2 expression in gastric epithelial dysplasia. ${ }^{15-17}$

Gastrointestinal epithelia produce site-specific mucins,${ }^{18}$ and the cell differentiation and lineage of various epithelia have been evaluated in various pathologic conditions based on the core mucin protein expression. ${ }^{19-21}$ In normal gastric mucosa, MUC5AC localizes on the surface/foveolar epithelium, whereas MUC6 is expressed in the mucous cells of the neck zone of oxyntic mucosa and antral glands. The intestinal mucin MUC2 and CD10, a marker of intestinal brush border differentiation, are not expressed in normal gastric mucosa. ${ }^{19,20}$ However, in previous studies, we and others have shown that gastric dysplasia and carcinoma show variable patterns of expression of these phenotypic markers. $^{22}$

The aim of this study was, therefore, to evaluate CDX2 expression in all stages of the metaplasiadysplasia-early-advanced gastric cancer continuum and correlate it with the morphological appearance and mucin phenotype.

\section{Materials and methods}

\section{Clinical Characteristics}

A consecutive series of 69 gastric endoscopic mucosal resections performed for a diagnosis of gastric epithelial dysplasia, 86 cases of intestinaltype early gastric cancer, and 56 cases of intestinaltype advanced gastric cancer, resected at Pusan National University Hospital, Busan, Korea between January 2004 and December 2004, was evaluated. All the specimens, obtained from the Korea Biobank, PNUH, were routinely fixed in $10 \%$ buffered formalin, embedded in paraffin, serially sectioned at $5 \mu \mathrm{m}$, and then stained with hematoxylin-eosin. For each case, clinical characteristics (age and sex, size, and location) were recorded, and for gastric epithelial dysplasia, several endoscopic features (ie, color, contour, and multicentricity) were also noted.

\section{Classification of Gastric Epithelial Dysplasia}

Each dysplastic lesion was classified as either adenomatous, foveolar, or hybrid type according to the previously described criteria. $^{23-25}$ In brief, adenomatous-type gastric epithelial dysplasia resembles colonic adenomas and is composed of large tubules lined by basophilic columnar cells with hyperchromatic, pencillate nuclei with pseudostratification, and a dense eosinophilic cytoplasm. Goblet cells and Paneth cells are commonly observed in this subtype (Figure 1). In contrast, foveolar gastric epithelial dysplasia shows either a hyperplastic foveolar region or small, irregular glands lined by tall columnar cells with pale cytoplasm and hyperchromatic round-ovoid nuclei. Glandular branching and epithelial intraluminal infoldings are often present. (Figure 2) Goblet cells and Paneth cells are rarely identified in this subtype. Cases of gastric epithelial dysplasia showing features of both foveolar and adenomatous dysplasia were classified as hybrid type. Each case was also graded as either low-grade or high-grade dysplasia according to the previously established criteria that included the degree of architectural complexity and cytological atypia ${ }^{3,24}$ (Figures 1 and 2).

\section{Immunohistochemical Analysis for Mucin Phenotype and CDX2}

Immunohistochemical stains for MUC5AC, MUC6, MUC2, CD10, and CDX2 were performed, and their expression was evaluated in dysplastic and adenocarcinomatous epithelium. The primary antibodies used in this study were summarized in Table 1.

In brief, $5-\mu \mathrm{m}$ thick consecutive sections were deparaffinized and hydrated through a graded series of alcohol. After antigen retrieval in $10 \mathrm{mmol} / \mathrm{l}$ citrate buffer ( $\mathrm{pH}$ 6.0) in a microwave oven for $10 \mathrm{~min}$, inhibition of endogenous peroxidase activity was performed by immersion in a 3\% H2O2/ methanol solution. The sections were then incubated with the primary antibodies followed by thorough washing in phosphate-buffered solution (PBS), incubation with the biotinylated secondary antibody, followed by the avidin-biotinylated horseradish peroxidase complex (Vectastain Elite ABC kit, Vector Laboratories30, Burlingame, CA, USA), and finally developed using DAB (3,3'-diaminobenzidine tetrachloride) as the chromogen. The nuclear counterstaining was accomplished using Mayer's hematoxylin.

To allow comparison with previously published data, only mucin immunoreactivity of at least $10 \%$ of the studied cell population was considered positive. Mucin phenotypes were further subdivided into gastric (only gastric mucin positive), gastrointestinal phenotypes (both gastric and intestinal mucin positive), intestinal phenotypes (only intestinal mucin positive), and null phenotypes (all mucin negative), based on the combination of predominant patterns of MUC5AC, MUC2, MUC6, and CD10 staining. Nuclear CDX2 staining was considered positive and was evaluated for the percentage of positively stained neoplastic cells. The cutoff value for positive CDX2 staining was predetermined as nuclear staining in at least $25 \%$ of the neoplastic cells as in the previous studies. ${ }^{17}$

\section{Statistical Analysis}

Statistical calculations were performed using SSPS version 10.0 for Windows software (SPSS Inc., 

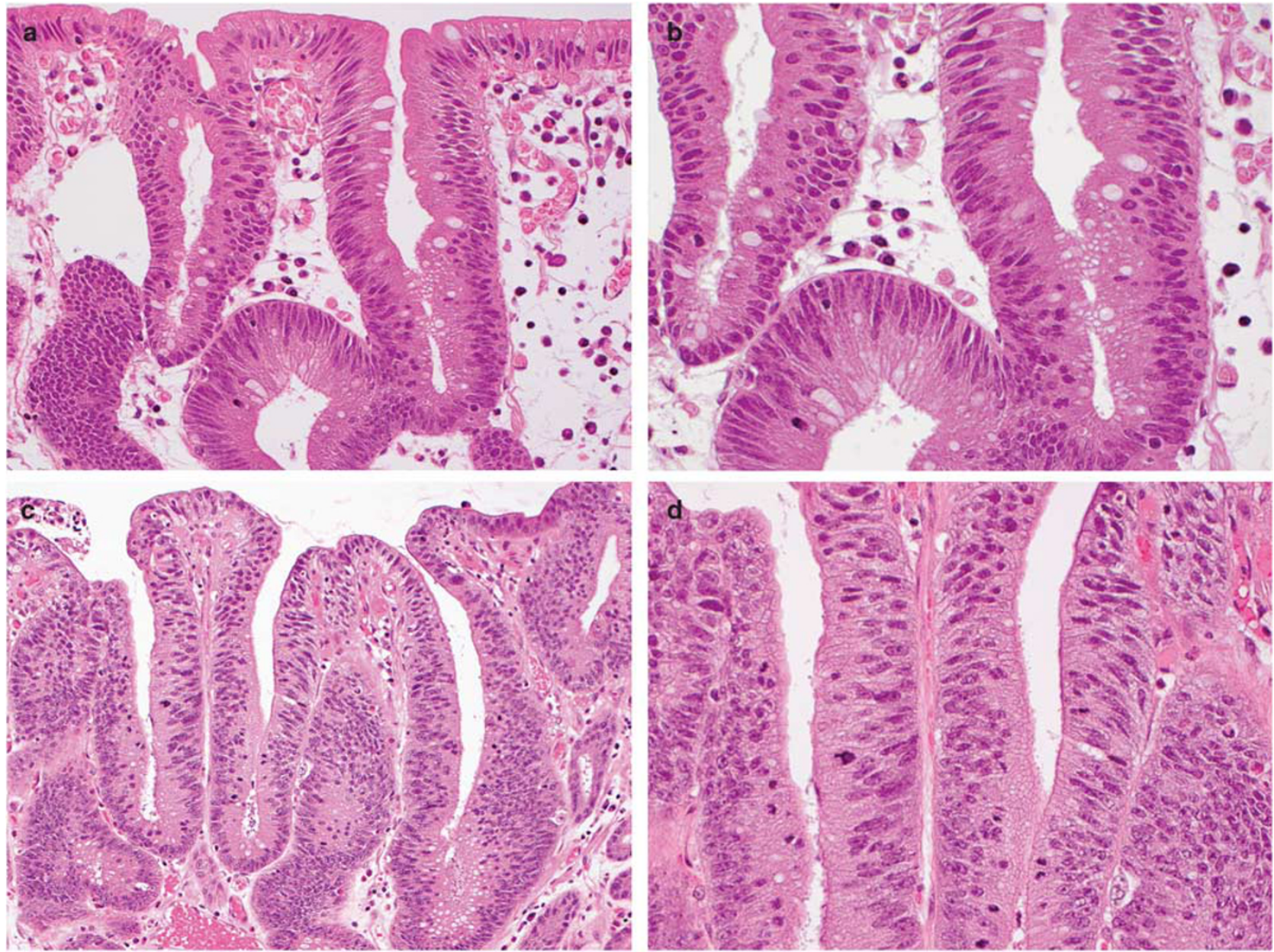

Figure 1 Examples of gastric epithelial dysplasia of the adenomatous (a and $\mathbf{b}$ ) and foveolar (c and $\mathbf{d}$ ) types.

Chicago, IL, USA). Fisher's probability exact test or $\chi^{2}$ test was used to test for observed differences between the study groups, and a $P<0.05$ was considered statistically significant.

\section{Results}

The gastric epithelial dysplasia group consisted of 44 men and 25 women, age range $27-81$ (mean: 63; s.d.: 9.6 years). The group of 86 early gastric cancers consisted of 69 men and 17 women. Their mean age was 60 (s.d.: 9.37; range: 34-76). The group of 56 advanced gastric cancers consisted of 46 men and 10 women. Their mean age was 61 (s.d.: 10.8; range: 32-84).

\section{Clinicopathological Characteristics and CDX2 Expression in Gastric Epithelial Dysplasia}

The size of gastric epithelial dysplasia ranged from 0.5 to $3.0 \mathrm{~cm}$ (mean: $1.3 \mathrm{~cm}$ ) (Table 2). With regard to morphotype, 15 cases were classified as foveolar, 31 as adenomatous, and 23 as hybrid gastric epithelial dysplasia. CDX2 expression was present in 44/69
(64\%) cases of gastric epithelial dysplasia. CDX2 expression was associated with the older age $(P=0.040)$ and with adenomatous- type gastric epithelial dysplasia, in which it was expressed in $87 \%$ of the cases. In contrast, a lower frequency of CDX2 expression was observed in the foveolar (7/15; $47 \%)$ and hybrid $(10 / 23 ; 44 \%)$ subtypes of gastric epithelial dysplasia, respectively $(P=0.001)$.

The foveolar and hybrid types of gastric epithelial dysplasia showed a downregulation of CDX2 expression when compared with the adjacent intestinal metaplasia $(21 / 38 ; 55 \%)$. This phenomenon was observed much less frequently in adenomatous gastric epithelial dysplasia $(4 / 31 ; 13 \% ; P=0.0002$, Figures 1 and 2). There was no statistically significant correlation of CDX2 expression in gastric epithelial dysplasia with the patient's sex, tumor size, location, endoscopic macroscopic appearance, and grade of dysplasia.

\section{Correlation of CDX2 Expression with Mucin Phenotypes in Gastric Epithelial Dysplasia}

A strong inverse correlation was observed between CDX2 expression and MUC5AC $(P=0.001$; Table 3$)$. 

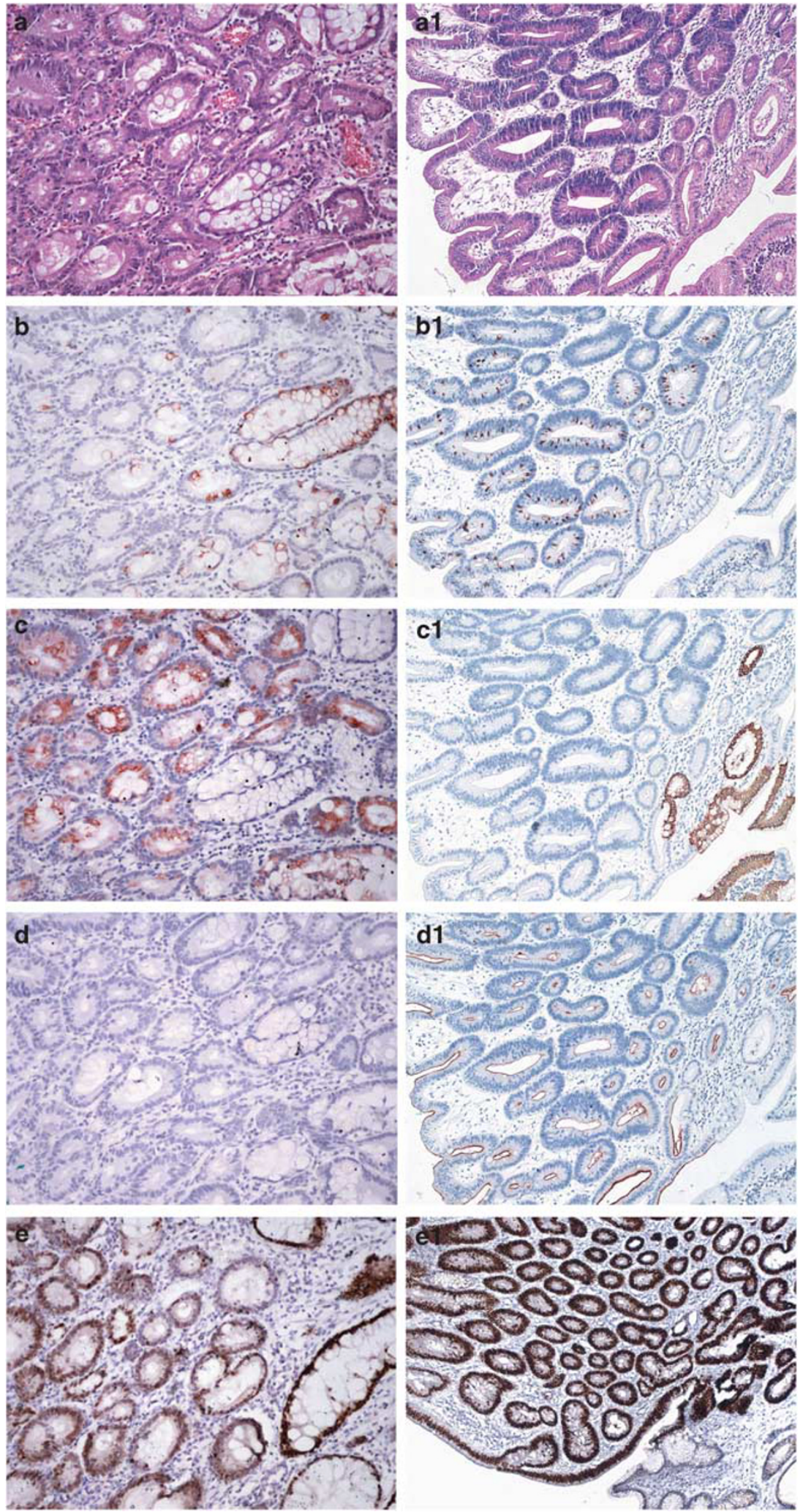

Figure 2 Immunophenotyping of foveolar (a) and adenomatous (b) gastric epithelial dysplasia. MUC2 (b, b1), scattered cells $(<10 \%)$ are positive in foveolar gastric epithelial dysplasia (b). MUC5AC (c, c1), adenomatous dysplasia is completely negative. CD10 (d, d1), complete absence of luminal staining in foveolar gastric epithelial dysplasia (d). CDX2 (e, e1), there is a decreased intensity of CDX2 expression in foveolar gastric epithelial dysplasia (e) compared with adenomatous gastric epithelial dysplasia (e1). 
Table 1 Primary antibodies used

\begin{tabular}{llc}
\hline $\begin{array}{l}\text { Primary antibodies } \\
\text { (clone) }\end{array}$ & Source & Dilution \\
\hline Muc2 (CLH2) & $\begin{array}{l}\text { Novocastra Laboratories, } \\
\text { Newcastle, UK }\end{array}$ & $1: 500$ \\
Muc5AC (CLH5) & Novocastra Laboratories & $1: 500$ \\
Muc6 (Ccp58) & Novocastra Laboratories & $1: 500$ \\
CD10 (56C6) & Novocastra Laboratories & $1: 100$ \\
CDX2 (CDX2-88) & Biogenex, San Ramon, CA, USA & $1: 100$ \\
\hline
\end{tabular}

Table 2 Characteristics of gastric epithelial dysplasia and CDX2 expression

\begin{tabular}{|c|c|c|c|}
\hline & \multicolumn{2}{|c|}{ CDX2 expression } & \multirow[t]{2}{*}{$\mathrm{P}$-value } \\
\hline & Negative $(\mathrm{n}=25)$ & Positive $(\mathrm{n}=44)$ & \\
\hline \multicolumn{4}{|l|}{ Age (year) } \\
\hline$<63$ & 13 & 12 & 0.04 \\
\hline$\geqslant 63$ & 12 & 32 & \\
\hline \multicolumn{4}{|l|}{ Sex } \\
\hline Male & 14 & 30 & 0.435 \\
\hline Female & 11 & 14 & \\
\hline \multicolumn{4}{|l|}{ Tumor size $(\mathrm{cm})$} \\
\hline$<1.4$ & 17 & 24 & 0.274 \\
\hline$\geqslant 1.4$ & 8 & 20 & \\
\hline \multicolumn{4}{|l|}{ Location } \\
\hline Body/fundus & 8 & 12 & 0.784 \\
\hline Antrum/pylorus & 17 & 32 & \\
\hline \multicolumn{4}{|l|}{ Gross type } \\
\hline Elevated & 21 & 34 & 0.756 \\
\hline Flat/depressed & 4 & 10 & \\
\hline \multicolumn{4}{|l|}{ Grade } \\
\hline Low & 9 & 18 & 0.688 \\
\hline High & 16 & 26 & \\
\hline \multicolumn{4}{|l|}{ Morphologic type } \\
\hline Foveolar & 8 & 7 & 0.001 \\
\hline Hybrid & 13 & 10 & \\
\hline Adenomatous & 4 & 27 & \\
\hline
\end{tabular}

In contrast, there was a positive correlation between CDX2 and CD10 expression $(P=0.005)$. No significant correlation was observed between CDX2 expression and the detection of MUC2 or MUC6 (Table 3).

\section{Correlation between CDX2 Expression, Mucin Expression, and the Depth of Invasion in the Intestinal-Type Gastric Carcinoma}

The rate of CDX2 expression was noted to decrease with the progressively increasing depth of invasion of gastric adenocarcinoma (Table 4). Observed in $63.8 \%$ of gastric epithelial dysplasia, CDX2 expression was noted in only $40 \%$ (35/88) of early type cancer and was even more decreased in advanced
Table 3 Correlation of CDX2 and mucin expression in gastric epithelial dysplasia

\begin{tabular}{|c|c|c|c|}
\hline & \multicolumn{2}{|c|}{ CDX2 expression } & \multirow[t]{2}{*}{ P-value } \\
\hline & Negative $(\mathrm{n}=25)$ & Positive $(\mathrm{n}=44)$ & \\
\hline \multicolumn{4}{|l|}{ Muc2 } \\
\hline$<10 \%$ & 20 & 30 & 0.291 \\
\hline$\geqslant 10 \%$ & 5 & 14 & \\
\hline \multicolumn{4}{|l|}{ Muc5AC } \\
\hline$<10 \%$ & 12 & 38 & 0.001 \\
\hline$\geqslant 10 \%$ & 13 & 6 & \\
\hline \multicolumn{4}{|l|}{ Muc6 } \\
\hline$<10 \%$ & 24 & 42 & 1.000 \\
\hline$\geqslant 10 \%$ & 1 & 2 & \\
\hline \multicolumn{4}{|l|}{ CD10 } \\
\hline$<10 \%$ & 25 & 33 & 0.005 \\
\hline$\geqslant 10 \%$ & 0 & 11 & \\
\hline
\end{tabular}

Table 4 CDX2 and mucin expression in gastric epithelial dysplasia and intestinal-type adenocarcinoma based on the depth of invasion

\begin{tabular}{|c|c|c|c|c|}
\hline & \multicolumn{3}{|c|}{ Depth of invasion } & \multirow[t]{2}{*}{$\mathrm{P}$-value } \\
\hline & $G E D(\mathrm{n}=69)$ & $E G C(\mathrm{n}=86)$ & $A G C(\mathrm{n}=56)$ & \\
\hline \multicolumn{5}{|c|}{ CDX2 positivity } \\
\hline Negative & 25 & 51 & 41 & 0.000 \\
\hline Positive & 44 & 35 & 15 & \\
\hline \multicolumn{5}{|c|}{ Muc2 positivity } \\
\hline Negative & 50 & 69 & 51 & 0.033 \\
\hline Positive & 19 & 17 & 5 & \\
\hline \multicolumn{5}{|c|}{ Mu5AC positivity } \\
\hline Negative & 50 & 66 & 43 & 0.794 \\
\hline Positive & 19 & 20 & 13 & \\
\hline \multicolumn{5}{|c|}{ Muc6 positivity } \\
\hline Negative & 66 & 82 & 55 & 0.654 \\
\hline Positive & 3 & 4 & 1 & \\
\hline \multicolumn{5}{|c|}{ CD10 positivity } \\
\hline Negative & 58 & 79 & 50 & 0.310 \\
\hline Positive & 11 & 7 & 6 & \\
\hline
\end{tabular}

GED, gastric epithelial dysplasia; EGC, early gastric cancer; AGC, advanced gastric cancer.

carcinomas (15/56, 27\%; Figure 3) MUC2 expression was also observed to decrease with the increasing depth of invasion. We did not observe any relationship between the expression of MUC5AC, MUC6, and CD10 and the depth of invasion.

\section{Correlation between CDX2 Expression and Mucin Phenotypes, Histologic}

A strong correlation was observed between CDX2 expression and MUC2 $(P=0.002)$. In contrast, there was a negative correlation between CDX2 and 


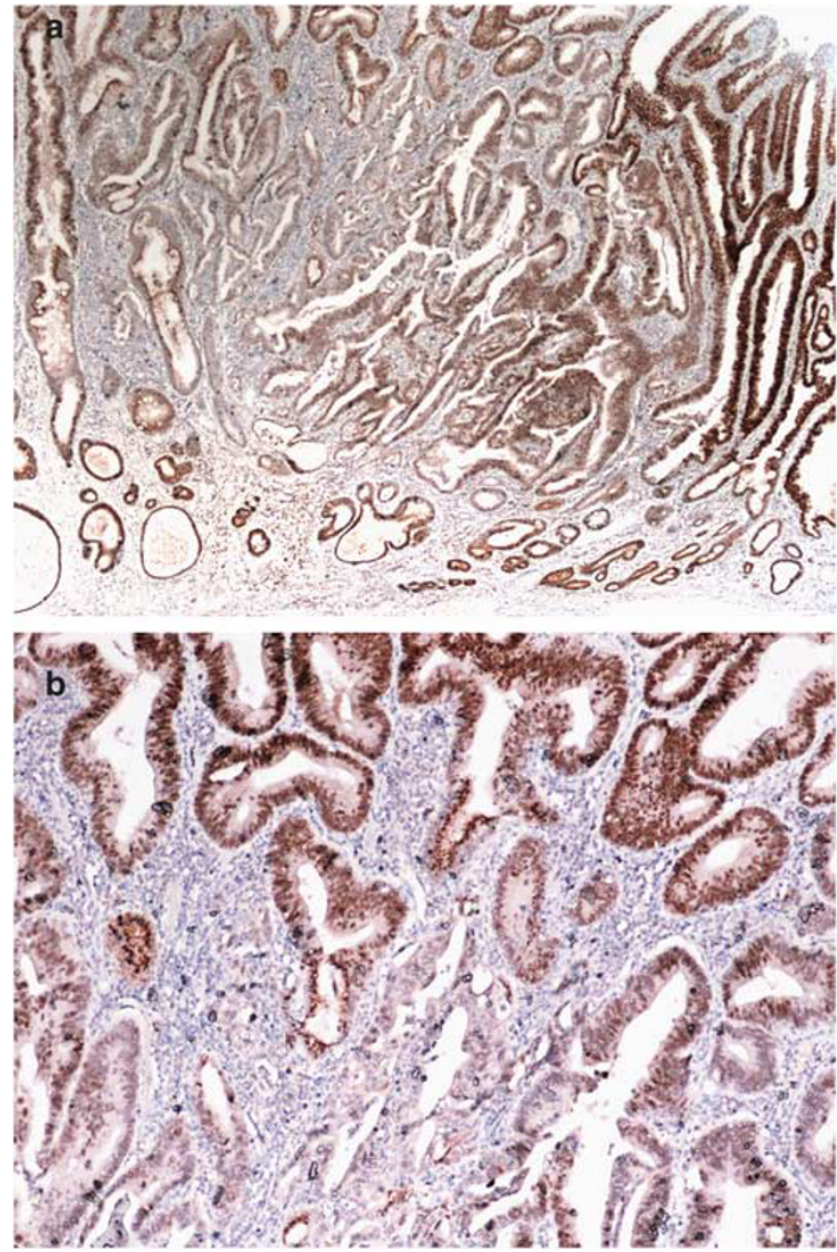

Figure 3 CDX2 immunostaining in the early stage of gastric cancer. The dysplastic component of the lesion (shoulders) shows preserved strong immunostaining (a), whereas it is lost in the invasive component (b).

MUC5AC expression $(P=0.019)$. (Table 5) No significant correlation was observed between CDX2 expression and the detection of MUC6 or CD10 (Table 5). With regard to the mucin phenotype, CDX2 expression was higher in the intestinal type $(27 / 53,51 \%)$ and the gastrointestinal type $(15 / 46$, $33 \%)$ as compared with the gastric type $(7 / 30,23 \%)$ and the null type $(1 / 13,8 \%)$.

\section{Correlation between CDX2 Expression, Histological Grade, Nodal Metastasis, and the Stage}

A strong inverse correlation was observed between CDX2 expression and nodal metastasis as well as the stage of intestinal-type gastric adenocarcinomas (Table 6). The decrease in CDX2 expression was associated with nodal metastasis $(P=0.015)$ and also an increase in the tumor stage $(P=0.016)$. No significant correlation was observed between CDX2 expression and the histological grade (Table 6).
Table 5 CDX2, mucin phenotypes, and mucin expression in the intestinal-type adenocarcinoma

\begin{tabular}{|c|c|c|c|}
\hline & \multicolumn{2}{|c|}{$C D X 2$} & \multirow[t]{2}{*}{$\mathrm{P}$-value } \\
\hline & Negative $(\mathrm{n}=92)$ & Positive $(\mathrm{n}=50)$ & \\
\hline \multicolumn{4}{|c|}{ Muc2 positivity } \\
\hline Negative & 84 & 36 & 0.002 \\
\hline Positive & 8 & 14 & \\
\hline \multicolumn{4}{|c|}{ Muc5AC positivity } \\
\hline Negative & 65 & 44 & 0.019 \\
\hline Positive & 27 & 6 & \\
\hline \multicolumn{4}{|c|}{ Muc6 positivity } \\
\hline Negative & 89 & 48 & 0.819 \\
\hline Positive & 3 & 2 & \\
\hline \multicolumn{4}{|c|}{ CD10 positivity } \\
\hline Negative & 85 & 44 & 0.386 \\
\hline Positive & 7 & 6 & \\
\hline \multicolumn{4}{|c|}{ Mucin Phenotype } \\
\hline G type & 23 & 7 & $0.001^{\mathrm{a}}$ \\
\hline GI type & 31 & 15 & \\
\hline I type & 26 & 27 & \\
\hline Null type & 12 & 1 & \\
\hline
\end{tabular}

G type, gastric type; GI type, gastrointestinal type; I type, intestinal type.

${ }^{\mathrm{a}}$ Between $\mathrm{G}$ type+null type versus I type+GI type.

Table 6 The relationship between CDX2 expression and lymph node metastasis, tumor grade, and the stage

\begin{tabular}{lccc}
\hline & \multicolumn{2}{c}{ CDX2 } & \multirow{2}{*}{ P-value } \\
\cline { 2 - 3 } & Negative (n=92) & Positive (n=50) & \\
\hline Lymph nodes & & & \\
$\quad$ Negative & 54 & 40 & 0.015 \\
$\quad$ Positive & 38 & 10 & \\
& & & \\
Histologic grade & & 20 & 0.263 \\
$\quad$ Well & 27 & 30 & \\
$\quad$ Moderate & 65 & & \\
& & 40 & 0.016 \\
Stage & & 4 & \\
I & 55 & 5 & \\
II & 13 & 1 & \\
III & 18 & & \\
IV & 6 & &
\end{tabular}

${ }^{\mathrm{a}}$ Between stage I and stages II+III+IV.

\section{Discussion}

In this study, we found that CDX2 expression is closely associated with adenomatous-type gastric epithelial dysplasia. We also observed that with the increasing expression of CDX2, the expression of CD10 increases and that of MUC5AC decreases. These findings affirm the association of CDX2 with an intestinal morphologic subtype and specific 
mucin phenotypes of gastric epithelial dysplasia. To the best of our knowledge, our study is the first to report directly on the correlation of CDX2 expression with morphological subtypes and mucin phenotypes of gastric epithelial dysplasia. We also observed an inverse correlation between CDX2 expression and the depth of invasion in the intestinal-type gastric adenocarcinomas.

Previous reports about the relationship between mucin expression and nuclear CDX2 reactivity showed that CDX2 expression is associated with the intestinal-type mucins (CD10 or MUC2). However, these studies were focused primarily on gastric adenocarcinomas. ${ }^{12,13}$ There are a few reports about CDX2 expression in gastric epithelial dysplasia, but the results are conflicting. For instance, Kim et $a l^{15}$ reported that $73.3 \%$ of low-grade adenomas and $85.5 \%$ of high-grade adenomas showed CDX2 expression. In their study, a significant reduction in CDX2 expression was also observed in the foci of gastric epithelial dysplasia when compared with the adjacent metaplastic gastric mucosa. In contrast, Liu et $a l^{16}$ reported a lower CDX2 expression in highgrade adenomas compared with low-grade adenomas and suggested that mucin expression in gastric epithelial dysplasias is not associated with CDX2 expression. In yet another study, with results contradictory to those mentioned above, Rugge et $a l^{17}$ reported that CDX2 expression is present in all cases of gastric epithelial dysplasia irrespective of the grade. In addition, no reduction in CDX2 expression in gastric epithelial dysplasia, as compared with adjacent intestinal metaplasia, was observed in their cases. ${ }^{17}$ These conflicting results may be partially explained by the use of different cutoff values to define CDX2 positivity (range $5-25 \%$ ), but we believe that they are largely a result of the lack of sub-typing gastric epithelial dysplasia.

Similar to Rugge's ${ }^{17}$ finding, we showed no relationship between the grade of gastric epithelial dysplasia and CDX2 expression. Not surprisingly, we also found that adenomatous gastric epithelial dysplasia shows greater CDX2 expression when compared with the foveolar type, and that an intestinal-type mucin marker, such as CD10, shows a positive correlation with CDX2 expression in gastric epithelial dysplasia. In our experience, CDX2 expression is decreased in the foveolar and hybrid types of dysplasia compared with the adenomatous type of dysplasia and the adjacent intestinal metaplasia.

In this study, CDX2 expression was decreased in early gastric cancers, when compared with dysplasia, and was even more reduced in advanced cancers. Similarly, Kim et $a l^{12}$ reported lesser CDX2 expression in early gastric cancers compared with advanced tumors. Mizoshita et $a l^{13}$ and Seno et $a 1^{14}$ also reported that CDX2 expression was associated with a favorable outcome. This collective experience may suggest a potential tumor suppressor role for CDX2, in view of its sequential decrease in expression along the stepwise gastric carcinogenesis (intestinal metaplasia, epithelial dysplasia, and early and advanced gastric cancer). This opinion is shared by Liu, who showed that CDX2 expression is progressively decreased in gastric intestinal metaplasia, dysplasia, and cancer. ${ }^{16}$ Interestingly, the progressive loss of CDX2 expression in Barrett's esophagus-associated dysplasia and adenocarcinoma, compared with non-dysplastic metaplastic epithelium, suggests a similar role in that setting. ${ }^{26}$

Experimentally, the report that mice with heterozygous disruption of CDX2 gene develop colonic polyps also suggests a tumor suppressor role. ${ }^{27}$ Furthermore, in vitro, CDX2 overexpression inhibits the growth of colon cancer cells. ${ }^{28}$

In conclusion, CDX2 has a significant role in gastric carcinogenesis. While ectopic CDX2 expression in mice induces gastric intestinal metaplasia, ${ }^{29}$ our current results, as well as those of others, lend further credence to the hypothesis that it also has a tumor suppressor role, especially with regard to progression into an advanced stage of gastric cancer. $^{30}$

\section{Disclosure/conflict of interest}

The authors declare no conflict of interest.

\section{References}

1 Stadtlander CT, Waterbor JW. Molecular epidemiology, pathogenesis and prevention of gastric cancer. Carcinogenesis 1999;20:2195-2208.

2 Laurén P. The two histologic main types of gastric carcinoma: diffuse and so-called intestinal-type carcinoma. An attempt at a histo-clinical classification. Acta Pathol Microbiol Scand 1965;64:31-49.

3 Schlemper RJ, Riddell RH, Kato Y, et al. The Vienna classification of gastrointestinal epithelial neoplasia. Gut 2000;47:251-255.

4 Yuasa Y. Control of gut differentiation and intestinaltype gastric carcinogenesis. Nat Rev Cancer 2003;3:592-600.

5 Beck F, Chawengsaksophak K, Waring $\mathrm{P}$, et al. Reprogramming of intestinal differentiation and intercalary regeneration in Cdx2 mutant mice. Proc Natl Acad Sci USA 1999;96:7318-7323.

6 Silberg DG, Swain GP, Suh ER, et al. Cdx1 and cdx2 expression during intestinal development. Gastroenterology 2000;119:961-971.

7 Bai YQ, Miyake S, Iwai T, et al. CDX2, a homeobox transcription factor, upregulates transcription of the p21/WAF1/CIP1 gene. Oncogene 2003;22:7942-7949.

8 Freund JN, Domon-Dell C, Kedinger M, et al. The Cdx1 and Cdx-2 homeobox genes in the intestine. Biochem Cell Biol 1998;76:957-969.

9 Mallo GV, Soubeyran P, Lissitzky JC, et al. Expression of the Cdx1 and Cdx2 homeotic genes leads to reduced malignancy in colon cancer-derived cells. J Biol Chem 1998;273:14030-14036.

10 Mallo GV, Rechreche H, Frigerio JM, et al. Molecular cloning, sequencing and expression of the mRNA 
encoding human Cdx1 and Cdx2 homeobox. Downregulation of $\mathrm{Cdx} 1$ and $\mathrm{Cdx} 2$ mRNA expression during colorectal carcinogenesis. Int J Cancer 1997;74:35-44.

11 Vider BZ, Zimber A, Hirsch D, et al. Human colorectal carcinogenesis is associated with deregulation of homeobox gene expression. Biochem Biophys Res Commun 1997;232:742-748.

12 Ha Kim G, Am Song G, Youn Park D, et al. CDX2 expression is increased in gastric cancers with less invasiveness and intestinal mucin phenotype. Scand J Gastroenterol 2006;41:880-886.

13 Mizoshita T, Tsukamoto T, Nakanishi H, et al. Expression of Cdx2 and the phenotype of advanced gastric cancers: relationship with prognosis. J Cancer Res Clin Oncol 2003;129:727-734.

14 Seno H, Oshima M, Taniguchi MA, et al. CDX2 expression in the stomach with intestinal metaplasia and intestinal-type cancer: prognostic implications. Int J Oncol 2002;21:769-774.

15 Kim HS, Lee JS, Freund JN, et al. CDX-2 homeobox gene expression in human gastric carcinoma and precursor lesions. J Gastroenterol Hepatol 2006;21:438-442.

$16 \mathrm{Liu} \mathrm{Q}$, Teh $\mathrm{M}$, Ito $\mathrm{K}$, et al. CDX2 expression is progressively decreased in human gastric intestinal metaplasia, dysplasia and cancer. Mod Pathol 2007; 20:1286-1297.

17 Rugge $\mathrm{M}$, Ingravallo G, Farinati $\mathrm{F}$, et al. Re: CDX2 homeotic gene expression in gastric noninvasive neoplasia. Am J Surg Pathol 2004;28:834-835.

18 Van Klinken BJ, Dekker J, Buller HA, et al. Biosynthesis of mucins (MUC2-6) along the longitudinal axis of the human gastrointestinal tract. Am J Physiol 1997;273: G296-G302.

19 Jass JR, Walsh MD. Altered mucin expression in the gastrointestinal tract: a review. J Cell Mol Med 2001;5:327-351.

20 Jass JR. Mucin core proteins as differentiation markers in the gastrointestinal tract. Histopathology 2000;37: 561-564.

21 Corfield AP, Myerscough N, Longman R, et al. Mucins and mucosal protection in the gastrointestinal tract: new prospects for mucins in the pathology of gastrointestinal disease. Gut 2000;47:589-594.

22 Machado JC, Nogueira AM, Carneiro F, et al. Gastric carcinoma exhibits distinct types of cell differentiation: an immunohistochemical study of trefoil peptides (TFF1 and TFF2) and mucins (MUC1, MUC2, MUC5AC, and MUC6). J Pathol 2000;190: 437-443.

23 Abraham SC, Montgomery EA, Singh VK, et al. Gastric adenomas: intestinal-type and gastric-type adenomas differ in the risk of adenocarcinoma and presence of background mucosal pathology. Am J Surg Pathol 2002;26:1276-1285.

24 Lewin KJ, Appleman HD. Carcinoma of the stomach In: Tumors of the Esophagus and Stomach. Atlas of Tumor Pathology (Third Series Fascicle 18) Armed Forces Institute of Pathology: Washington, DC, 1996, pp 245-330.

25 Park do Y, Srivastava A, Kim GH, et al. Adenomatous and foveolar gastric dysplasia: distinct patterns of mucin expression and background intestinal metaplasia. Am J Surg Pathol 2008;32:524-533.

26 Phillips RW, Frierson Jr HF, Moskaluk CA. Cdx 2 as a marker of epithelial intestinal differentiation in the esophagus. Am J Surg Pathol 2003;27:1442-1447.

27 Chawengsaksophak K, James R, Hammond VE, et al. Homeosis and intestinal tumours in Cdx2 mutant mice. Nature 1997;386:84-87.

28 Mallo GV, Soubeyran P, Lissitzky JC, et al. Expression of the $\mathrm{Cdx} 1$ and $\mathrm{Cdx} 2$ homeotic genes leads to reduced malignancy in colon cancer-derived cells. J Biol Chem 1998;273:14030-14036.

29 Silberg DG, Sullivan J, Kang E, et al. Cdx2 ectopic expression induces gastric intestinal metaplasia in transgenic mice. Gastroenterology 2002;122: 689-696.

30 Tajima Y, Yamazaki K, Makino R, et al. Gastric and intestinal phenotypic marker expression in early differentiated-type tumors of the stomach: clinicopathologic significance and genetic background. Clin Cancer Res 2006;12:6469-6479. 